\title{
Linitis plástica: implicación pronóstica actual de un concepto clásico
}

\author{
Linitis plastica: current prognostic implication of a classic concept
}

Aingeru Sarriugarte-Lasarte',2,3*, Eva García-Alberdi', Silvia Pérez-Fernández², Oihane Gutiérrez-Grijalba', Patricia Mifsut-Porcel', Yanina Kataryniuk-Di Costanzo', Patricia Sendino-Cañizares' e Iratxe Rodeño-Esteban ${ }^{1}$ ${ }^{1}$ Servicio de Cirugía General y Aparato Digestivo, Hospital Universitario de Cruces; ${ }^{2}$ Cirugía Computacional, Biocruces Bizkaia; ${ }^{3}$ Departamento de Cirugía, Universidad del País Vasco (UPV/EHU); ${ }^{4}$ Servicio de Anatomía Patológica, Hospital Universitario de Cruces. Barakaldo, Vizcaya, España

\section{Resumen}

Antecedentes: $A$ pesar de haber sido descrita hace varios siglos, sigue sin estar clara la implicación pronóstica de la linitis plástica (LP). Objetivo: Analizar el impacto de la LP en la supervivencia de los pacientes intervenidos de gastrectomía por adenocarcinoma gástrico. Método: Estudio retrospectivo unicéntrico de los casos de $L P$ diagnosticados en nuestro centro durante 5 años. Resultados: Se agruparon en LP, carcinomas difusos no LP (DNLP) y resto de adenocarcinomas (ADC). En el análisis comparativo se incluyeron 199 gastrectomías realizadas en el mismo periodo de tiempo. Con una mediana de seguimiento de 54.5 meses (intervalo de confianza del 95\% [IC 95\%]: 37.0-65.0), la supervivencia mediana de la LP fue de 14 meses (IC 95\%: 9-27), significativamente menor ( $p=0.002)$ que en los DNLP, que fue de 51.5 meses (IC 95\%: 25-70), y que en el resto de los ADC, en los que fue de 62 meses (IC 95\%: 43-68). La supervivencia global a 1, 3 y 5 años fue estadísticamente peor en el grupo de LP (69, 15 y 8\%) comparado con los grupos de DNLP (82, 54 y 44\%) y ADC (89, 63 y 54\%) $(p=0.073, p<0.001$ y $p<0.001$, respectivamente). En el análisis multivariante se identificó la LP como un factor de riesgo independiente de supervivencia (hazard ratio: 3.26; $p=0.001$ ). Conclusiones: La LP es un factor pronóstico independiente asociado a mayor mortalidad por cáncer gástrico.

Palabras clave: Cáncer gástrico. Linitis plástica. Pronóstico.

\begin{abstract}
Background: Despite having been described for several centuries linitis plastica's (LP) prognostic implication remain unclear. Objective: To analyze the impact of $L P$ on the survival of patients undergoing gastrectomy for gastric adenocarcinoma. Method: A single-center retrospective study of cases of $L P$ diagnosed and confirmed by pathological anatomy has been carried out in a third-level center for 5 years. Results: They were grouped into Linitis Plastica $(L P)$, diffuse non-LP carcinomas $(D N L P)$ and other adenocarcinomas (ADC). 199 gastrectomies performed in the same period of time were included in the comparative analysis. With a median follow-up of 54.5 months (95\% Cl: 37.0-65.0), the median survival of LP was 14 months (95\% Cl: 9-27) significantly lower ( $p=0.002)$ compared to DNLP 51.5 months (95\% Cl: 25-70) and the rest of ADC 62 months (95\% Cl: 43-68). The OS at 1,3 and 5 years was statistically worse in the LP group (69, 15, 8\%) compared to DNLP (82, 54, 44\%) and ADC (89, 63, 54\%) $(p=0.073, p<0.001, p<0.001)$. In the multivariate analysis, LP was identified as an independent risk factor for OS with an $H R$ of $3.26(p=0.001)$. Conclusions: $L P$ is an independent prognostic factor associated with higher mortality in gastric cancer.
\end{abstract}

Keywords: Gastric cancer. Linitis plastica. Prognostic.

\section{Correspondencia:}

*Aingeru Sarriugarte-Lasarte

Lukieta 3

Fecha de recepción: 09-10-2020

C.P. 48100, Mungia, Bizkaia, España

E-mail: aingeru.sarriugartelasarte@osakidetza.eus

0009-7411/@ 2021 Academia Mexicana de Ciru

(http://creativecommons.org/licenses/by-nc-nd/4.0/).

Fecha de aceptación: 28-02-2021

DOI: 10.24875/CIRU.20001106
Cir Cir. 2022;90(1):64-73

Contents available at PubMed www.cirugiaycirujanos.com jo la licencia CC BY-NC-ND 


\section{Introducción}

Se conoce como linitis plástica (LP) al aspecto macroscópico engrosado, rígido y sin aparente ulceración mucosa que presentan los órganos a los que afecta un carcinoma de tipo difuso. Conocido y descrito desde hace varios siglos, se ha asociado habitualmente a tumores gástricos; no obstante, aunque excepcionalmente, puede presentarse en otras localizaciones, como la colorrectal, sea como tumor primario o secundario metastásico ${ }^{1}$. Se trata de un proceso incierto y enigmático desde el punto de vista patológico y clínico, pero sobre todo se desconoce claramente su pronóstico y cuál debe ser la actitud terapéutica ${ }^{2}$. Con el objeto de valorar la implicación pronóstica que dicho proceso presenta en nuestro centro, se ha realizado un estudio de supervivencia con los casos detectados.

\section{Método}

\section{Población de estudio}

Se han estudiado todas las gastrectomías con intención curativa realizadas durante 5 años (2010 a 2014) en un hospital terciario. Por otro lado, se han analizado los casos de LP detectados por valoración macroscópica intraoperatoria y confirmación anatomopatológica de las muestras durante el mismo periodo de tiempo. Se han excluido los casos con sospecha preoperatoria por prueba de imagen no confirmada en vivo. Se ha realizado un estudio descriptivo de los casos de LP y se han comparado con los casos de cáncer gástrico resecados en el mismo periodo de tiempo.

\section{Definición}

Se define como LP el carcinoma, habitualmente de localización gástrica, que a causa de la infiltración difusa de las células tumorales produce un engrosamiento circunferencial de la pared gástrica, que sin presentar necesariamente masa tumoral conlleva una rigidez de la pared.

\section{Características clínico-patológicas}

Se resecaron con intención curativa 199 casos de cáncer gástrico, de los cuales 63 (32.8\%) fueron de tipo difuso y $13(6.5 \%)$ de ellos presentaron LP. En el mismo periodo de tiempo, el servicio de anatomía patológica confirmó un total de 25 casos de LP. No obstante, fueron excluidos del análisis comparativo los tumores resecados sin intención curativa y los de localización extragástrica: tres casos de cáncer de localización colorrectal, solo uno de ellos de localización primaria rectal y dos como metástasis a distancia de cáncer gástrico difuso en el contexto del proceso conocido como «escudo de Blumer»3; dos de localización esofágica primaria, y otro de localización en el intestino delgado como metástasis de cáncer gástrico difuso.

\section{Tipo de resección tumoral}

El objetivo principal de la cirugía fue lograr la extirpación completa del tumor (resección R0), aunque en los casos de irresecabilidad o diseminación peritoneal no se realizó resección o se llevaron a cabo procedimientos paliativos (R2) para tratar las complicaciones relacionadas con el tumor. Los casos se clasificación por estadios y ganglios linfáticos, según la clasificación patológica pTNM (8. ${ }^{a}$ ed.) del American Joint Committee on Cancer (AJCC).

\section{Análisis de los datos}

Las variables categóricas se describen mediante frecuencias y porcentajes, y las variables continuas mediante la media y la desviación típica, en caso de seguir una distribución normal, o mediante la mediana y el rango intercuartílico en caso contrario. La comparación entre las variables categóricas se realizó con la prueba de ji al cuadrado o el test exacto de Fisher cuando las frecuencias esperadas eran $<5$. Para las variables continuas se usó la prueba $t$ de Student cuando seguían una distribución normal o el test $U$ de Mann-Whitney en caso contrario.

Las curvas de supervivencia se estimaron utilizando el método de Kaplan-Meier y se compararon mediante la prueba de log-rank, con el tiempo de supervivencia medido desde la fecha de diagnóstico por imagen o por biopsia hasta la fecha del fallecimiento o del último seguimiento. Para ver qué variables influyen en la supervivencia se realizó un análisis de regresión de Cox univariante, y aquellas variables con $p<0.200$ se incluyeron en un análisis de regresión de Cox multivariante. Se eliminaron las variables que perdían la significación estadística ( $p>0.05$ ), hasta quedarnos con el modelo que se presenta en 
el apartado de resultados. Estos se han expresado mediante hazard ratio (HR) y su intervalo de confianza del 95\% (IC 95\%).

Todos los análisis se realizaron con el programa estadístico R (versión 3.6.1) (https:// www.R-project.org/).

\section{Comité de ética}

Todos los pacientes dieron su consentimiento informado por escrito para que su información fuera utilizada con fines de investigación. Se obtuvo la aprobación del comité de ética del hospital para la realización del estudio, el cual se realizó de acuerdo con los estándares éticos de la Declaración de Helsinki de la Asociación Médica Mundial.

\section{Resultados}

\section{Tasa de resección}

Durante el periodo estudiado se han detectado 19 casos de LP de localización gástrica, de los cuales $13(68 \%)$ pudieron ser resecados con intención curativa. Todos los tumores se presentaron en estadio localmente avanzado T3 o T4 y N+ (estadios II/III). El $32 \%$ restante mostró como hallazgo intraoperatorio enfermedad a distancia M1, a modo de carcinomatosis peritoneal, por lo que no se realizó resección tumoral completa (R2). Únicamente el $61.5 \%$ de los tumores resecados pudo considerarse $\mathrm{R} 0$, ya que el $38.5 \%$ presentaron afectación microscópica del margen de resección (R1).

Los casos con resección paliativa recibieron quimioterapia paliativa y aquellos con resección Ro fueron tratados mediante quimioterapia y radioterapia con intención adyuvante.

De los 199 casos intervenidos con intención curativa presentaron LP el 6.5\%. De los pacientes con LP, el $76.9 \%$ eran hombres, con una edad media de 64.8 $( \pm 12.2)$ años. Todos los casos de LP fueron pobremente diferenciados o indiferenciados. Según la clasificación de Lauren, todos los casos de LP fueron de histología difusa, con lo que el $15.9 \%$ de todos los tumores difusos presentaron LP. Según la clasificación de la Organización Mundial de la Salud (OMS), el $38.5 \%$ de los casos de LP tuvieron histología de células en anillo de sello y el $61.5 \%$ fueron de tipo pobremente cohesivo. Por otro lado, destaca que el $100 \%$ de los casos de LP presentaron afectación gástrica transmural $\mathrm{T} 3 / \mathrm{T} 4$, frente al $65 \%$ del resto de los casos, y una muy importante carga ganglionar tumoral: N0 $7.69 \%$ vs. $30.1 \%$ y N3 $71.6 \%$ vs. $23.5 \%$, respectivamente.

La tabla 1 presenta las principales características demográficas y clínicas de la cohorte en estudio.

\section{Análisis de supervivencia}

La mediana de supervivencia de los pacientes con LP fue de 12 meses (IC 95\%: 1-54): 14 meses (IC 95\%: 9-27) en el grupo de tumores resecados (16,8 meses en los R0) y 3 meses en el de no resecados. El análisis univariado se refleja en los gráficos de Kaplan-Meier, mostrados en la figura 1, y en la tabla 2. La supervivencia a 1, 3 y 5 años de los casos de LP fue significativamente menor que en los grupos de tumores difusos sin LP y el resto de los adenocarcinomas. La supervivencia mediana de los pacientes con LP fue significativamente menor que la de aquellos con carcinoma gástrico difuso no LP o con el resto de los adenocarcinomas resecados en el mismo periodo de tiempo: 14 meses (IC 95\%: 9-27) vs. 51.5 meses (IC 95\%: 25-70) y 62 meses (IC 95\%: 43-68), respectivamente. En el análisis multivariante de supervivencia global, la presencia de LP se ha mostrado como un factor de riesgo independiente de mortalidad (HR: 3.26; IC 95\%: 1.58-6.72). Otros factores independientes de supervivencia global han sido la infiltración ganglionar $(N)(p<0.002)$, la linfadenectomía con más de 15 ganglios $(p<0.001)$, la resección tumoral completa $(R 0)(p<0.001)$ y el tratamiento adyuvante recibido $(p<0.013)$ (Tabla 3$)$. En un análisis multivariante realizado para valorar exclusivamente el grupo de carcinomas difusos, la LP también se muestra como un factor independiente de menor supervivencia global (HR: 2.97; IC 95\%: 1.49-5.94).

\section{Discusión}

\section{Anatomía patológica}

El término «linitis plástica» fue acuñado para describir el engrosamiento de la pared gástrica que produce esta patología, refiriéndose a la semejanza con las fibras de lino que la submucosa adquiere macroscópicamente $^{4}$. Con el tiempo, se ha utilizado para describir el aspecto macroscópico de un carcinoma con infiltración difusa que afecta cualquier órgano hueco, conservando su forma, pero quedando rígido 
Tabla 1. Características clínico-patológicas

\begin{tabular}{|c|c|c|c|c|}
\hline & $\begin{array}{l}\text { Todos } \\
(n=199)\end{array}$ & $\begin{array}{c}\text { No LP } \\
(n=186)\end{array}$ & $\begin{array}{l}\mathrm{LP} \\
(n=13)\end{array}$ & $p$ \\
\hline Edad, años & $68.2( \pm 11.8)$ & $68.5( \pm 11.8)$ & $64.8( \pm 12.2)$ & 0.315 \\
\hline $\begin{array}{l}\text { Edad categórica } \\
>65 \text { años }\end{array}$ & $123(61.8 \%)$ & 117 (62.9\%) & $6(46.2 \%)$ & 0.249 \\
\hline $\begin{array}{l}\text { Sexo } \\
\text { Hombres }\end{array}$ & $134(67.3 \%)$ & $124(66.7 \%)$ & $10(76.9 \%)$ & 0.552 \\
\hline $\begin{array}{l}\text { Localización } \\
\text { Tercio superior } \\
\text { Cuerpo } \\
\text { Antro }\end{array}$ & $\begin{array}{c}39(19.6 \%) \\
54(27.1 \%) \\
106(53.3 \%)\end{array}$ & $\begin{array}{c}33(17.7 \%) \\
50(26.9 \%) \\
103(55.4 \%)\end{array}$ & $\begin{array}{l}6(46.2 \%) \\
4(30.8 \%) \\
3(23.1 \%)\end{array}$ & 0.021 \\
\hline $\begin{array}{l}\text { Técnica } \\
\text { Gastrectomía total } \\
\text { Gastrectomía subtotal }\end{array}$ & $\begin{array}{c}60(31.6 \%) \\
130(68.4 \%)\end{array}$ & $\begin{array}{c}50(28.2 \%) \\
127(71.8 \%)\end{array}$ & $\begin{array}{c}10(76.9 \%) \\
3(23.1 \%)\end{array}$ & 0.001 \\
\hline $\begin{array}{l}\text { Histología Lauren } \\
\text { Indeterminado o mixto } \\
\text { Intestinal } \\
\text { Difuso }\end{array}$ & $\begin{array}{l}47(24.5 \%) \\
82(42.7 \%) \\
63(32.8 \%)\end{array}$ & $\begin{array}{l}47(26.3 \%) \\
82(45.8 \%) \\
50(27.9 \%)\end{array}$ & $\begin{array}{l}0(0.00 \%) \\
0(0.00 \%) \\
13(100 \%)\end{array}$ & $<0.001$ \\
\hline $\begin{array}{l}\text { Histología OMS } \\
\text { Indeterminado o mixto } \\
\text { Papilar/tubular } \\
\text { Células en anillo de sello } \\
\text { Mucinoso } \\
\text { Pobremente cohesivo }\end{array}$ & $\begin{array}{l}54(27.1 \%) \\
73(36.7 \%) \\
29(14.6 \%) \\
10(5.03 \%) \\
33(16.6 \%)\end{array}$ & $\begin{array}{l}54(29.0 \%) \\
73(39.2 \%) \\
24(12.9 \%) \\
10(5.38 \%) \\
25(13.4 \%)\end{array}$ & $\begin{array}{l}0(0.00 \%) \\
0(0.00 \%) \\
5(38.5 \%) \\
0(0.00 \%) \\
8(61.5 \%)\end{array}$ & $<0.001$ \\
\hline $\begin{array}{l}\text { T patológico } \\
\text { T1 } \\
\text { T2 } \\
\text { T3 } \\
\text { T4a/T4b }\end{array}$ & $\begin{array}{l}25(12.6 \%) \\
40(20.1 \%) \\
94(47.2 \%) \\
40(20.1 \%)\end{array}$ & $\begin{array}{l}25(13.4 \%) \\
40(21.5 \%) \\
88(47.3 \%) \\
33(17.7 \%)\end{array}$ & $\begin{array}{l}0(0.00 \%) \\
0(0.00 \%) \\
6(46.2 \%) \\
7(53.8 \%)\end{array}$ & 0.008 \\
\hline $\begin{array}{l}\text { N patológico } \\
\text { N0 } \\
\text { N1 } \\
\text { N2 } \\
\text { N3a } \\
\text { N3b }\end{array}$ & $\begin{array}{l}56(28.6 \%) \\
37(18.9 \%) \\
52(26.5 \%) \\
38(19.4 \%) \\
13(6.63 \%)\end{array}$ & $\begin{array}{l}55(30.1 \%) \\
37(20.2 \%) \\
48(26.2 \%) \\
32(17.5 \%) \\
11(6.01 \%)\end{array}$ & $\begin{array}{l}1(7.69 \%) \\
0(0.00 \%) \\
4(30.8 \%) \\
6(46.2 \%) \\
2(15.4 \%)\end{array}$ & 0.011 \\
\hline $\begin{array}{l}\text { Linfadenectomía D } \\
\text { Paliativa }(<15) \\
\text { D1 } \\
\text { D2 }\end{array}$ & $\begin{array}{c}11(5.64 \%) \\
113(57.9 \%) \\
71(36.4 \%)\end{array}$ & $\begin{array}{c}11(6.04 \%) \\
105(57.7 \%) \\
66(36.3 \%)\end{array}$ & $\begin{array}{l}0(0.00 \%) \\
8(61.5 \%) \\
5(38.5 \%)\end{array}$ & 1.000 \\
\hline $\begin{array}{l}\text { Resección ampliada } \\
\text { Sí }\end{array}$ & 16 (8.04\%) & $13(6.99 \%)$ & $3(23.1 \%)$ & 0.074 \\
\hline $\begin{array}{l}\text { Bordes anastomosis } \\
\text { Negativos } \\
\text { Positivos }\end{array}$ & $\begin{array}{l}175(88.8 \%) \\
22(11.2 \%)\end{array}$ & $\begin{array}{c}167(90.8 \%) \\
17(9.24 \%)\end{array}$ & $\begin{array}{l}8(61.5 \%) \\
5(38.5 \%)\end{array}$ & 0.008 \\
\hline $\begin{array}{l}\text { G } \\
\text { Bien diferenciado } \\
\text { Moderadamente diferenciado } \\
\text { Pobremente diferenciado o Indiferenciado }\end{array}$ & $\begin{array}{l}47(25.7 \%) \\
45(24.6 \%) \\
91(49.7 \%)\end{array}$ & $\begin{array}{l}47(27.6 \%) \\
45(26.5 \%) \\
78(45.9 \%)\end{array}$ & $\begin{array}{l}0(0.00 \%) \\
0(0.00 \%) \\
13(100 \%)\end{array}$ & $<0.001$ \\
\hline R 102 & $20(10.2 \%)$ & $15(8.15 \%)$ & $5(38.5 \%)$ & 0.005 \\
\hline $\begin{array}{l}\text { Adyuvancia } \\
\text { No } \\
\text { QT } \\
\text { QT-RT }\end{array}$ & $\begin{array}{l}71(45.2 \%) \\
23(14.6 \%) \\
63(40.1 \%)\end{array}$ & $\begin{array}{l}67(46.5 \%) \\
20(13.9 \%) \\
57(39.6 \%)\end{array}$ & $\begin{array}{l}4(30.8 \%) \\
3(23.1 \%) \\
6(46.2 \%)\end{array}$ & 0.447 \\
\hline
\end{tabular}


Tabla 1. Características clínico-patológicas (Continuación)

\begin{tabular}{|c|c|c|c|c|}
\hline & $\begin{array}{c}\text { Todos } \\
(n=199)\end{array}$ & $\begin{array}{c}\text { No LP } \\
(n=186)\end{array}$ & $\begin{array}{c}\text { LP } \\
(n=13)\end{array}$ & $p$ \\
\hline $\begin{array}{l}\text { Recidiva } \\
\text { Sí }\end{array}$ & $103(52.8 \%)$ & 90 (49.5\%) & $13(100 \%)$ & 0.001 \\
\hline $\begin{array}{l}\text { Estadio TNM }\left(8 .^{\text {a } e d .)}\right. \\
\text { IA } \\
\text { IB } \\
\text { IIA } \\
\text { IIB } \\
\text { IIIA } \\
\text { IIIB } \\
\text { IIIC }\end{array}$ & $\begin{array}{l}18(9.28 \%) \\
23(11.9 \%) \\
28(14.4 \%) \\
25(12.9 \%) \\
51(26.3 \%) \\
37(19.1 \%) \\
12(6.19 \%)\end{array}$ & $\begin{array}{l}18(9.94 \%) \\
23(12.7 \%) \\
27(14.9 \%) \\
25(13.8 \%) \\
47(26.0 \%) \\
31(17.1 \%) \\
10(5.52 \%)\end{array}$ & $\begin{array}{l}0(0.00 \%) \\
0(0.00 \%) \\
1(7.69 \%) \\
0(0.00 \%) \\
4(30.8 \%) \\
6(46.2 \%) \\
2(15.4 \%)\end{array}$ & 0.055 \\
\hline
\end{tabular}

LP: linitis plástica; OMS: Organización Mundial de la Salud; QT: quimioterapia; RT: radioterapia.

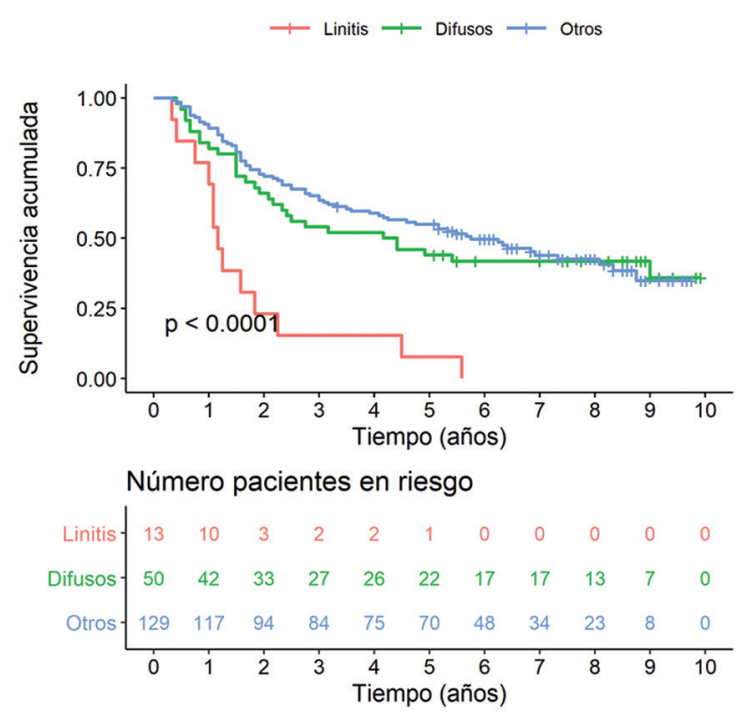

Figura 1. Graficas de Kaplan-Meier de supervivencia global.

y contraído, como una bota de cuero. Microscópicamente se caracteriza por el aumento de tejido conectivo en la capa submucosa, con escasa celularidad pobremente cohesiva y en algunas ocasiones en forma de anillo de sello ${ }^{5}$.

Aunque habitualmente hace referencia a tumores gástricos, en ocasiones la LP puede afectar a otras localizaciones del tracto digestivo y urinario, presentándose como metástasis de tumores gástricos 0 de mama $^{6}$. Se han descrito LP secundarias en localización colorrectal y excepcionalmente como metástasis tardía, años tras el diagnóstico, en localización intestinal ${ }^{7,8}$. No obstante, también existen algunos casos en la literatura de linitis rectal primaria por adenocarcinoma de células de anillo de sello, representando menos del $0.1 \%$ de los tumores colorrectales ${ }^{9-13}$.
Tabla 2. Supervivencia global

\begin{tabular}{lcccc}
\hline Supervivencia global & LP & Difusos & No LP ni difuso & $\mathbf{p}$ \\
\hline A 1 año & $69 \%$ & $82 \%$ & $89 \%$ & 0.073 \\
A 3 años: & $15 \%$ & $54 \%$ & $63 \%$ & $<0.001$ \\
A 5 años & $8 \%$ & $44 \%$ & $54 \%$ & $<0.001$ \\
Mediana, meses & $14(9-27) 51.5(25-70)$ & $62(43-68)$ & 0.002 \\
\hline LP: linitis plástica.
\end{tabular}

Es complejo identificar la LP dentro de los subtipos de las clasificaciones histopatológicas de cáncer gástrico, pero casi siempre se ha reportado como morfología macroscópica de los estadios avanzados de dicha enfermedad, sin ser realmente equivalente a ninguno de estos. El tipo IV de la clasificación de Bormann hace referencia al tipo infiltrativo y difuso que produce engrosamiento gástrico sin afectación mucosa y con margen tumoral incierto ${ }^{14,15}$. Microscópicamente se relaciona con el carcinoma escirro de la clasificación japonesa, clasificado por su abundante estroma formado por colágeno I y III, secretado por fibroblastos y miofibroblastos modulados por las escasas células cancerosas, dentro del proceso conocido como reacción desmoplásica ${ }^{16}$. En la clasificación de Lauren, el carcinoma de tipo difuso es el que más presenta morfología de LP, y dentro de la clasificación de la OMS suele considerarse dentro de los tumores poco cohesivos, incluyendo los tumores con células en anillo de sello y en raras ocasiones los carcinomas mucinosos. Aparentemente, tal como ocurre en el cáncer gástrico difuso hereditario ligado al gen $\mathrm{CDH} 1$, la desregulación de las moléculas de adhesión intercelular E-cadherinas produce la desunión celular, 
Tabla 3. Análisis univariante y multivariante de la supervivencia global

\begin{tabular}{|c|c|c|c|c|c|c|}
\hline & \multicolumn{3}{|c|}{ Análisis univariante } & \multicolumn{3}{|c|}{ Análisis multivariante } \\
\hline & HR & (IC 95\%) & p & HR & (IC 95\%) & $\mathrm{p}$ \\
\hline Edad, años & 1.02 & $(1.00-1.03)$ & 0.028 & 1 & $(0.98-1.03)$ & 0.7 \\
\hline Sexo, mujeres & 0.93 & $(0.63-1,35)$ & 0.689 & 1.33 & $(0.85-2.09)$ & 0.213 \\
\hline LP AP, sí & 4.11 & $(2.28-7.39)$ & $<0.001$ & 3.26 & $(1.58-6.72)$ & 0.001 \\
\hline $\begin{array}{l}\text { Localización (ref. cardias) } \\
\text { Cuerpo } \\
\text { Antro }\end{array}$ & $\begin{array}{l}0.59 \\
0.67\end{array}$ & $\begin{array}{l}(0.35-1.00) \\
(0.43-1.05)\end{array}$ & $\begin{array}{l}0.106 \\
0.049 \\
0.080\end{array}$ & & & \\
\hline $\begin{array}{l}\text { Grado diferenciación (ref. 1) } \\
2 \\
3-4\end{array}$ & $\begin{array}{l}1.86 \\
2.56\end{array}$ & $\begin{array}{l}(1.02-3.38) \\
(1.52-4.32)\end{array}$ & $\begin{array}{c}0.001 \\
0.041 \\
<0.001\end{array}$ & & & \\
\hline $\begin{array}{l}\text { T patológico_rec1 (ref. T1) } \\
2 \\
3 \\
4\end{array}$ & $\begin{array}{l}1.48 \\
3.74 \\
7.93\end{array}$ & $\begin{array}{l}(0.60-3.64) \\
(1.71-8.17) \\
(3.49-18.0)\end{array}$ & $\begin{array}{c}<0.001 \\
0.389 \\
0.001 \\
<0.001\end{array}$ & & & \\
\hline $\begin{array}{l}\text { N patológico rec (ref. N0) } \\
\text { N patológico: N1 } \\
\text { N patológico: N2 } \\
\text { N patológico: N3 }\end{array}$ & $\begin{array}{l}1.56 \\
3.28 \\
5,13\end{array}$ & $\begin{array}{l}(0.82-2.96) \\
(1.92-5.60) \\
(2.07-12.7)\end{array}$ & $\begin{array}{c}<0.001 \\
0.173 \\
<0.001 \\
<0.001\end{array}$ & $\begin{array}{l}3.44 \\
4.33\end{array}$ & $\begin{array}{l}(1.57-7.54) \\
(2.01-9.33)\end{array}$ & $\begin{array}{l}0.002 \\
<0.001\end{array}$ \\
\hline Linfadenectomía D: D1 & 0.29 & $(0.15-0.56)$ & $<0.001$ & 0.15 & $(0.07-0.33)$ & $<0.001$ \\
\hline Linfadenectomía D: D2 & 0.28 & $(0.14-0.56)$ & $<0.001$ & 0.14 & $(0.06-0.31)$ & $<0.001$ \\
\hline $\begin{array}{l}\mathrm{R} \text { (ref. Ro) } \\
102\end{array}$ & 3,01 & $(1.84-4.91)$ & $\begin{array}{l}<0.001 \\
<0.001\end{array}$ & 3.62 & $(1.92-6.81)$ & $<0.001$ \\
\hline $\begin{array}{l}\text { Adyuvancia (ref. No) } \\
\text { QT } \\
\text { QT-RT }\end{array}$ & $\begin{array}{l}0.76 \\
0.49\end{array}$ & $\begin{array}{l}(0.44-1.31) \\
(0.32-0.74)\end{array}$ & $\begin{array}{l}0,003 \\
0.318 \\
0.001\end{array}$ & $\begin{array}{l}0.43 \\
0.31\end{array}$ & $\begin{array}{l}(0.22-0.84) \\
(0.18-0.54)\end{array}$ & $\begin{array}{l}0.013 \\
<0.001\end{array}$ \\
\hline
\end{tabular}

AP: anatomía patológica; HR: hazard ratio; IC 95\%: intervalo de confianza del 95\%; LP: linitis plástica; QT: quimioterapia; RT: radioterapia.

favoreciendo la migración y la infiltración tumoral. Por otro lado, las nuevas clasificaciones moleculares basadas en el comportamiento biológico de los tumores permiten clasificar más concretamente, sin grupos indeterminados, los diferentes tipos de tumores gástricos en relación con la genómica y la epigenética, orientando hacia la posible detección de dianas terapéuticas $^{17}$. No obstante, aunque posiblemente la mayor parte de los casos de LP se engloben dentro del grupo estable genómicamente, muy relacionado así mismo con el cáncer gástrico difuso, ninguna de las clasificaciones hace clara referencia a la LP.

En la literatura existen escasas series y con pocos casos que definan y analicen por separado los pacientes con LP dentro de los pacientes con cáncer gástrico tratados con intención curativa ${ }^{2}$. Además, son muy diversas las definiciones, tanto preoperatorias como intraoperatorias o posoperatorias, utilizadas para fijar los casos, por lo que los grupos resultan muy diversos. En nuestro estudio se clasificaron como LP los casos con hallazgo intraoperatorio de engrosamiento circunferencial de la pared intestinal, habitualmente gástrica, que conlleva su rigidez, y con posterior confirmación anatomopatológica de la afectación tumoral difusa con reacción desmoplásica sin presencia necesaria de masa tumoral mucosa (Fig. 2).

Tal como se expone en nuestro estudio, la literatura también muestra que la LP afecta en su mayoría a hombres algo más jóvenes que aquellos con cáncer gástrico en general. La LP se presenta de manera silente, sin producir sintomatología hasta estadios avanzados, en los que la disminución de la distensibilidad gástrica puede producir náuseas y vómitos, por lo que el diagnóstico precoz resulta complejo. La endoscopia y la biopsia son el método de referencia en el diagnóstico del cáncer gástrico ${ }^{18}$. No obstante, en los casos de LP, las células pueden difundir por la submucosa hasta convertirse con rapidez en un tumor 


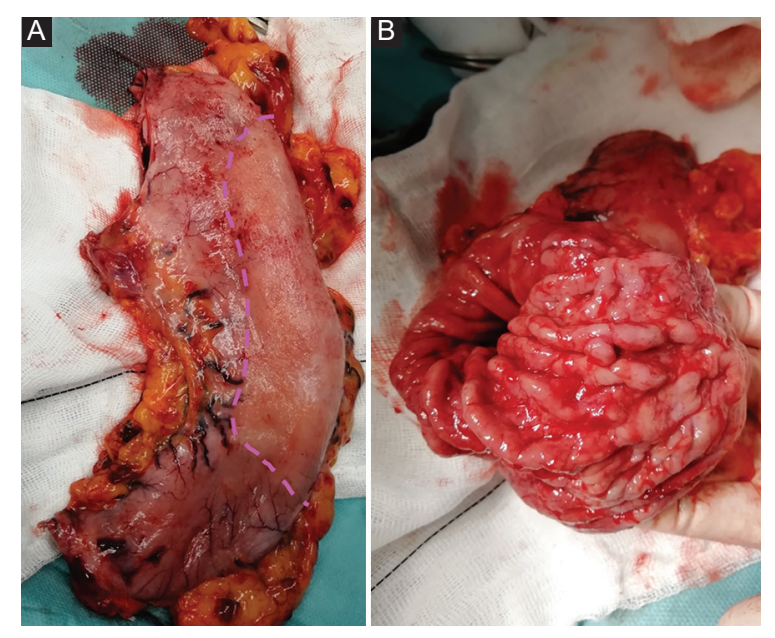

Figura 2. A: pieza quirúrgica en la que se aprecia el engrosamiento mural gástrico producido por la linitis plástica. B: pieza quirúrgica en la que se aprecia normalidad de la mucosa.

transmural, respetando hasta en el $30 \%$ de los casos la mucosa intacta ${ }^{19}$. En las ocasiones en que no se aprecia ulceración en la mucosa, esta puede mostrarse como una superficie plana de aspecto atrófico o como pliegues engrosados causados por la contracción submucosa. Por lo tanto, la LP puede afectar solo a una parte del estómago, produciendo una menor distensibilidad de ese segmento, y progresivamente ir extendiéndose a todo el estómago ${ }^{20}$. Aunque la literatura muestra que puede ser diagnosticada desde estadios iniciales (T1-2), la mayor parte de los casos son diagnosticados en estadios avanzados de infiltración local (T3-4 > 70\%). En nuestro estudio, la presentación como un tumor transmural desde el diagnóstico ha supuesto el $100 \%$ de los casos, lo que probablemente está en relación con la definición que ha servido como criterio de inclusión.

Por otro lado, la escasa celularidad del tumor comparada con el abundante estroma puede conllevar un importante número de biopsias negativas (30-36\%), por lo que es preciso un alto grado de sospecha clínica para realizar biopsias aleatorias o guiadas por ecoendoscopia que, aunque con posibles falsos negativos, conducirán al diagnóstico ${ }^{21}$. La biopsia óptica in vivo mediante microscopía confocal se encuentra en desarrollo y podría ser de utilidad para el diagnóstico temprano de la LP22.

En caso de sospecharse la presencia de LP preoperatoriamente, bien por la identificación en pruebas de imagen (tránsito baritado, tomografía computarizada - resonancia magnética) o por la visualización directa por endoscopia o ecoendoscopia, con o sin confirmación anatomopatológica en biopsias, se debe valorar la realización de una laparoscopia exploradora con lavado intraoperatorio y toma de macrobiopsias, pues al igual que en nuestro estudio $(32 \%)$ varias series han mostrado la coexistencia de carcinomatosis peritoneal hasta en el $64 \%$ de los casos $^{23}$. No obstante, en muchas ocasiones la primera sospecha de LP es intraoperatoria, por observarse en el acto quirúrgico una zona gástrica con apariencia sospechosa, sin tener previamente un diagnóstico anatomopatológico de tumor indiferenciado, lo que dificulta la toma de decisiones en lo que al tratamiento respecta. Por otro lado, se han descrito metástasis a distancia con el mismo perfil de infiltración difusa, en ocasiones de neoplasias de células en anillo de sello gástricas o de mama en colon, recto o intestino delgado, incluso años después del diagnóstico de la enfermedad ${ }^{9}$.

Diversos estudios han buscado biomarcadores en sangre periférica, así como células circulantes, cDNA o miRNA, pero posiblemente la escasa celularidad de estos tumores no ha permitido encontrar un método diagnóstico estandarizable ${ }^{24,25}$. Los futuros análisis proteómicos sobre tejido de biopsias o muestras sanguíneas podrían llegar a un diagnóstico preoperatorio más temprano y certero, con el fin de conseguir personalizar el tratamiento y mejorar el pronóstico.

En nuestra serie, un importante número de casos de LP se localizaron en el tercio superior (46.2\%) del estómago, y con el objetivo de lograr unos márgenes amplios de resección, la gastrectomía total fue la técnica más utilizada $(76.9 \%)$, al igual que se reporta en la literatura (62.5-88\%). Aunque algunos autores llegan a dudar de la posibilidad de ofrecer tratamiento quirúrgico curativo en los casos de LP, clásicamente se han propuesto unos márgenes de seguridad amplios $(5-8 \mathrm{~cm})$ con el objetivo de conseguir una resección $\mathrm{R} 0$ en los tumores con infiltración difusa ${ }^{26}$. No obstante, algunos estudios han intentado probar que no son necesarios unos márgenes tan amplios para la resección de este tipo de tumores ${ }^{27}$. Ante la ausencia específica de estudios que analicen el valor de los márgenes de seguridad en caso de LP, se recomienda resecar un margen de seguridad lo suficientemente amplio que evite la resección R1, evaluándolo mediante secciones intraoperatorias congeladas, sin necesidad de realizar de entrada una gastrectomía total a todos los pacientes con LP, con el fin de evitar la morbimortalidad que esta conlleva. Además, la gastrectomía total sistemática tampoco garantiza la extirpación $\mathrm{R} 0$ del tumor en la pieza quirúrgica, ya que los 
límites de infiltración son macroscópicamente imprecisos y pueden sobrepasar microscópicamente la unión esofagogástrica hacia el esófago. En nuestro caso, el 38.5\% presentaron márgenes positivos (R1), cifra acorde con la literatura, en la que se reportan altas tasas de resección insuficiente (31-69\%). Por lo tanto, hay que ser cauto con las resecciones limitadas, ya que en ocasiones se han descrito falsos negativos de los márgenes de resección por congelación causados por la baja celularidad de los tumores escirros con reacción desmoplásica. Posiblemente, la gastrectomía casi total o del $95 \%$, con biopsia intraoperatoria de los márgenes quirúrgicos, podría cumplir las condiciones de resección en algunos casos, aunando las ventajas de los amplios márgenes de seguridad y las bajas tasas de morbimortalidad posoperatoria de la gastrectomía subtotal ${ }^{28}$. Dicha resección, junto con una linfadenectomía D2 completa, podría ser el estándar de tratamiento del cáncer gástrico con LP resecable.

Ante el mayor número de casos de implantes peritoneales microscópicos, técnicas como la omentectomía completa y la bursectomía toman mayor importancia en el caso de la LP, pudiendo estar recomendadas en tumores $\mathrm{T} 3 / \mathrm{T} 4{ }^{29}$. Por otro lado, en los últimos años ha comenzado a desarrollarse la quimioterapia intraoperatoria (HIPEC, hyperthermic intraperitoneal chemotherapy) en el cáncer gástrico ${ }^{30}$. Aunque por el momento ningún estudio ha descrito específicamente su utilidad en la LP, esta técnica resulta esperanzadora debido a la importancia de la diseminación peritoneal de estos tumores, por lo que se están llevando a cabo nuevos estudios ${ }^{31}$.

De la misma manera, cuando la LP se presenta en otra localización distinta de la gástrica, en caso de lesiones primarias se recomienda la resección tumoral en bloque con márgenes amplios y linfadenectomía extensa. No obstante, la mayoría de las veces se trata de lesiones secundarias metastásicas de un tumor primario gástrico o de mama, por lo que el tratamiento se realizará con carácter paliativo $0^{6-13}$.

El tratamiento neoadyuvante del cáncer gástrico presenta varias teóricas ventajas, como un mejor control local y una menor tasa de resecciones $\mathrm{R} 1$, y al tratarse en general de tumores localmente avanzados en el momento del diagnóstico podría considerarse en muchos casos de LP. No obstante, los resultados de diferentes esquemas quimioterápicos, desde el MAGIC hasta el actual FLOT, parecen mostrar una menor respuesta de los tumores de tipo infiltrante difuso ${ }^{32,33}$. Por lo tanto, aunque algunos estudios proponen la cirugía radical de entrada basándose en la posible futilidad de estos tratamientos y la progresión de la enfermedad durante su aplicación, probablemente presenten un perfil de quimiosensibilidad distinto, por lo que se están realizando nuevos estudios para determinar la utilidad de los tratamientos adyuvantes en este tipo de tumores $^{34}$. En nuestra serie, el tratamiento más utilizado en los pacientes con LP fue la quimioterapia y radioterapia posoperatoria según el esquema de Mc Donald (46.2\%), que es el tratamiento más utilizado en los tumores localmente avanzados previo a la utilización de las terapias neoadyuvantes actualmente más aceptadas.

Por otro lado, la mayoría de los tumores que pueden producir LP son negativos para HER-2, por lo que la terapia dirigida con trastuzumab no está indicada. Los futuros ensayos sobre proteómica deben dirigirse a detectar posibles dianas terapéuticas, no solo celulares, sino también en el abundante estroma que presentan los tumores con LP, de modo que puedan aplicarse terapias personalizadas.

\section{Pronóstico}

Varios estudios han tratado de establecer el pronóstico de los tumores que pueden producir LP, pero las diferentes definiciones de esta hacen que los resultados sean heterogéneos. La supervivencia media es de 8 a 13.8 meses, llegando a 16.7 meses en los pacientes con tumores resecados ${ }^{2}$. Del mismo modo que se observa en nuestra serie, la LP parece tener características pronósticas diferentes de los casos sin LP: estadios más avanzados en el momento del diagnóstico; mayor infiltración linfática, con gran carga ganglionar y diseminación peritoneal, pero con menor tasa de metástasis hepáticas; mayor tasa de resecciones R1 (31-69\%) con positividad de márgenes quirúrgicos a pesar de una mayor tasa de gastrectomía total (62.5$100 \%)$; y mayor morbimortalidad perioperatoria. No obstante, aunque algunos grupos refieren que la LP no difiere en cuanto a pronóstico del resto de los cánceres gástricos si se ajusta por estadios ${ }^{35,36}$, en nuestro estudio se ha mostrado como factor de riesgo independiente de mortalidad global por cáncer (HR: 3.26; IC 95\%: 1.58-6.72). Incluso realizando un análisis multivariado teniendo en cuenta solo los tumores difusos, la LP se muestra como un factor independiente de mal pronóstico (HR: 2.97; IC 95\%: 1.49-5.94).

\section{Conclusiones}

La LP es un concepto antiguo que ha llegado hasta el día de hoy en diferentes clasificaciones 
histopatológicas sin que realmente exista una clara definición de ella. Se trata de una afección macroscópica, en la que se aprecia un órgano rígido y de paredes engrosadas, con expresión microscópica, causado por tumores con baja celularidad de escasa cohesión entre sí y abundante estroma intercelular que presenta una infiltración difusa de los tejidos afectos. A pesar de que la gran mayoría de las veces afecta al estómago de manera primaria, puede aparecer en todo el tubo digestivo y se ha descrito en localización colorrectal e intestinal, bien como origen de la enfermedad o como metástasis de carcinomas poco cohesivos de otras localizaciones.

Se trata de un proceso incierto desde el punto de vista tanto terapéutico como pronóstico. En este estudio, la LP se ha mostrado como un factor independiente relacionado con la mortalidad por cáncer. Por lo tanto, es preciso un alto grado de sospecha clínica para posibilitar un diagnóstico precoz de esta insidiosa patología, que permita un tratamiento con intención curativa. En este sentido, la cirugía debe seguir proponiéndose como parte de un tratamiento multidisciplinario potencialmente curativo del cáncer gástrico que produce LP. No obstante, son necesarias más herramientas diagnósticas, como marcadores biológicos, que permitan tomar decisiones terapéuticas individualizadas y modificar las estrategias quirúrgicas, así como detectar dianas terapéuticas dentro del contexto de la medicina personalizada.

\section{Agradecimientos}

Especial agradecimiento al Dr. Oleagoitia Cilaurre, jefe de la Sección de Cirugía Esófago-Gástrica del Hospital de Cruces, recién jubilado.

\section{Conflicto de intereses}

Los autores declaran no tener ningún conflicto de intereses.

\section{Responsabilidades éticas}

Protección de personas y animales. Los autores declaran que los procedimientos seguidos se conformaron a las normas éticas del comité de experimentación humana responsable y de acuerdo con la Asociación Médica Mundial y la Declaración de Helsinki.
Confidencialidad de los datos. Los autores declaran que han seguido los protocolos de su centro de trabajo sobre la publicación de datos de pacientes.

Derecho a la privacidad y consentimiento informado. Los autores han obtenido el consentimiento informado de los pacientes y/o sujetos referidos en el artículo. Este documento obra en poder del autor de correspondencia.

\section{Bibliografía}

1. Brinton W. The diseases of the stomach, with an introduction on its anatomy and physiology; being lectures delivered at St. Thomas's hospital. London: J. Churchill; 1859.

2. Agnes A, Estrella JS, Badgwell B. The significance of a nineteenth century definition in the era of genomics: linitis plastica. World J Surg Oncol. 2017;15:123.

3. Haubrich, WS. Blumer of Blumer's shelf. Gastroenterology. 2000;118:30.

4. IARC Publications. Digestive SystemTumours. World Health Organization. $5^{\text {th }}$ ed. Volume 1 . Chapter 3 . Tumours of the stomach. Lyon, Francia. 2019.

5. Nagtegaal ID, Odze RD, Klimstra D, et al. The 2019 WHO classification of tumours of the digestive system. Histopathology. 2020;76(2):182-188. doi: $10.1111 /$ his. 13975

6. Dixon CF. Carcinoma of linitis plastica type involving intestine. Ann Surg. 1936;103:263-72.

7. Katsinelos P, Papaziogas B, Chatzimavroudis G, Katsinelos T, Dimou E, Atmatzidis $S$, et al. Secondary rectal linitis plastica as first manifestation of urinary bladder carcinoma. Ann Gastroenterol. 2012;25:173-5.

8. Fahl JC, Dockerty MB, Judd ES. Scirrhous carcinoma of the colon and rectum. Surg Gynecol Obstet. 1960;111:759-66.

9. Rodríguez Ortega M, Carabias Hernández A, Rodríguez Barbero JM, Montano Navarro E, Limones Esteban M. Linitis plástica intestinal, metástasis tardía de adenocarcinoma gástrico en anillo de sello. Rev Esp Enferm Dig. 2006;98:391-2.

10. Laufman $\mathrm{H}$, Saphir O. Primary linitis plastica type carcinoma the colon. Arch Surg. 1951;62:79-91.

11. Murayama $H$, Miura K, Sakamoto J. Primary linitis plastica of the rectum: report of four cases and review of the literature. Surg Today. 1996;26: 875-81.

12. Nadel L, Mori K, Shinya H. Primary linitis plastica of the colon and rectum. Dis Colon Rectum. 183;26:738-42.

13. Domínguez-Adame E. Linitis plástica primaria rectal: presentación de un caso clínico con abordaje quirúrgico laparoscópico. Cirugía. Casos Clínicos. 2008;3:2-7.

14. Japanese Gastric Cancer Association. Japanese gastric cancer treatment guidelines 2018 ( ${ }^{5} \mathrm{~h}$ edition). Gastric Cancer. 2021;24(1):1-21.

15. Hu B, El Hajj N, Sittler S, Lammert N, Barnes R, Meloni-Ehrig A. Gastric cancer: classification, histology and application of molecular pathology. $J$ Gastrointest Oncol. 2012;3:251-61.

16. Minamoto T, Ooi A, Okada Y, Mai M, Nagai Y, Nakanishi I. Desmoplastic reaction of gastric carcinoma: a light- and electron-microscopic immunohistochemical analysis using collagen type-specific antibodies. Hum Pathol. 1988;19:815-21.

17. Bass AJ, Thorsson V, Shmulevich I, Reynolds SM, Miller M, Bernard B, et al.; Cancer Genome Atlas Research Network. Comprehensive molecular characterization of gastric adenocarcinoma. Nature. 2014;513: 202-9.

18. Smyth EC, Verheij M, Allum W, Cunningham D, Cervantes A, Arnold D. Gastric cancer: ESMO Clinical Practice Guidelines for diagnosis, treatment and follow-up. Ann Oncol. 2016;27:v38-v49.

19. Jung K, Park MI, Kim SE, Park SJ. Borrmann type 4 advanced gastric cancer: focus on the development of scirrhous gastric cancer. Clin Endosc. 2016;49:336-45.

20. Endo K, Sakurai M, Kusumoto E, Uehara H, Yamaguchi S, Tsutsumi N, et al. Biological significance of localized type IV scirrhous gastric cancer. Oncol Lett. 2012;3:94-9.

21. Park MS, Ha HK, Choi BS, Kim KW, Myung SJ, Kim AY, et al. Scirrhous gastric carcinoma: endoscopy versus upper gastrointestinal radiography. Radiology. 2004;231:421-6.

22. Goetz M, Watson A, Kiesslich R. Confocal laser endomicroscopy in gastrointestinal diseases. J Biophotonics. 2011;4:498-508.

23. Kodera $Y$, Nakanishi $H$, Ito S, Mochizuki Y, Yamamura Y, Fujiwara M, et al. Detection of disseminated cancer cells in linitis plastica-type gastric carcinoma. Jpn J Clin Oncol. 2004;34:525-31. 


\section{A. Sarriugarte-Lasarte et al.: Linitis plástica}

24. Ichikawa Y, Koshikawa N, Hasegawa S, Ishikawa T, Momiyama N, Kunizaki $\mathrm{C}$, et al. Marked increase of trypsin(ogen) in serum of linitis plastica (gastric cancer, borrmann 4) patients. Clin Cancer Res. 2000:6:1385-8

25. Dakubo GD. Cancer biomarkers in body fluids. Switzerland: Springer 2017. 509 p

26. Japanese Gastric Cancer Association. Japanese gastric cancer treatment guidelines 2014 (ver. 4). Gastric Cancer. 2017;20:1-19.

27. Squires $3^{\text {rd }} \mathrm{MH}$, Kooby DA, Poultsides GA, Pawlik TM, Weber SM, Schmidt $\mathrm{CR}$, et al. Is it time to abandon the $5-\mathrm{cm}$ margin rule during resection of distal gastric adenocarcinoma? A multi-institution study of the U.S. Gastric Cancer Collaborative. Ann Surg Oncol. 2015;22: 1243-51.

28. Azagra JS, Sarriugarte A, Ibáñez FJ. Estado actual de la gastrectomía por cáncer. «Less is often more». Cir Esp. 2018:96:603-5.

29. Marano L, Polom K, Bartoli A, Spaziani A, De Luca R, Lorenzon L, et al. Oncologic effectiveness and safety of bursectomy in patients with advanced gastric cancer: a systematic review and updated meta-analysis. $\mathrm{J}$ Invest Surg. 2018;31:529-38.

30. Yonemura Y, Canbay E, Li Y, Coccolini F, Glehen O, Sugarbaker PH et al. A comprehensive treatment for peritoneal metastases from gastric cancer with curative intent. Eur J Surg Oncol. 2016;42:1123-31.

31. Brown ZJ, Hernandez JM, Ripley, RT, Davis JL. Heated intraperitoneal chemotherapy and gastrectomy for gastric cancer in the U.S.: the time is now. J Gastrointest Oncol. 2017;8:1109-13.
32. Cunningham D, Allum WH, Stenning SP, Thompson JN, Van de Velde CJ, Nicolson M, et al. Perioperative chemotherapy versus surgery alone for resectable gastroesophageal cancer. $N$ Engl J Med. 2006;355:11-20.

33. Al-Batran SE, Hofheinz RD, Pauligk C, Kopp HG, Haag GM, Luley KB, et al. Histopathological regression after neoadjuvant docetaxel, oxaliplatin, fluorouracil, and leucovorin versus epirubicin, cisplatin, and fluorouracil or capecitabine in patients with resectable gastric or gastro-oesophageal junction adenocarcinoma (FLOT4-AIO): results from the phase 2 part of a multicentre, open-label, randomised phase 2/3 trial. Lancet Oncol. 2016;17:1697-708.

34. Piessen G, Messager M, Le Malicot K, Robb WB, Di Fiore F, Guilbert M, et al. Phase II/III multicentre randomised controlled trial evaluating a strategy of primary surgery and adjuvant chemotherapy versus peri-operative chemotherapy for resectable gastric signet ring cell adenocarcinomas - PRODIGE 19 - FFCD1103 - ADCI002. BMC Cancer. 2013;13:281.

35. Blackham AU, Swords DS, Levine EA, Fino NF, Squires MH, Poultsides G, et al. Is linitis plastica a contraindication for surgical resection: a multi- institution study of the U.S. Gastric Cancer Collaborative. Ann Surg Oncol. 2016;23:1203-11.

36. Thompson RJ, Ranaghan L, Kennedy R, Clements W, Carey PD, Kennedy JA. Survival following operative management of gastric linitis plastica compared with non-operative management. Ann R Coll Surg Engl. 2017:99:228-32. 